\title{
Urgences
}

\section{Autobiographie d'un style}

\section{Jacques Daignault}

Numéro 21, novembre 1988

Bagatelles et crases

URI : https://id.erudit.org/iderudit/025492ar

DOI : https://doi.org/10.7202/025492ar

Aller au sommaire du numéro

Éditeur(s)

Urgences

ISSN

0226-9554 (imprimé)

1927-3924 (numérique)

Découvrir la revue

Citer ce document

Daignault, J. (1988). Autobiographie d'un style. Urgences, (21), 21-44.

https://doi.org/10.7202/025492ar

Ce document est protégé par la loi sur le droit d'auteur. L'utilisation des services d'Érudit (y compris la reproduction) est assujettie à sa politique d'utilisation que vous pouvez consulter en ligne.

https://apropos.erudit.org/fr/usagers/politique-dutilisation/
Cet article est diffusé et préservé par Érudit.

Érudit est un consortium interuniversitaire sans but lucratif composé de l’Université de Montréal, l'Université Laval et l'Université du Québec à Montréal. Il a pour mission la promotion et la valorisation de la recherche. https://www.erudit.org/fr/ 


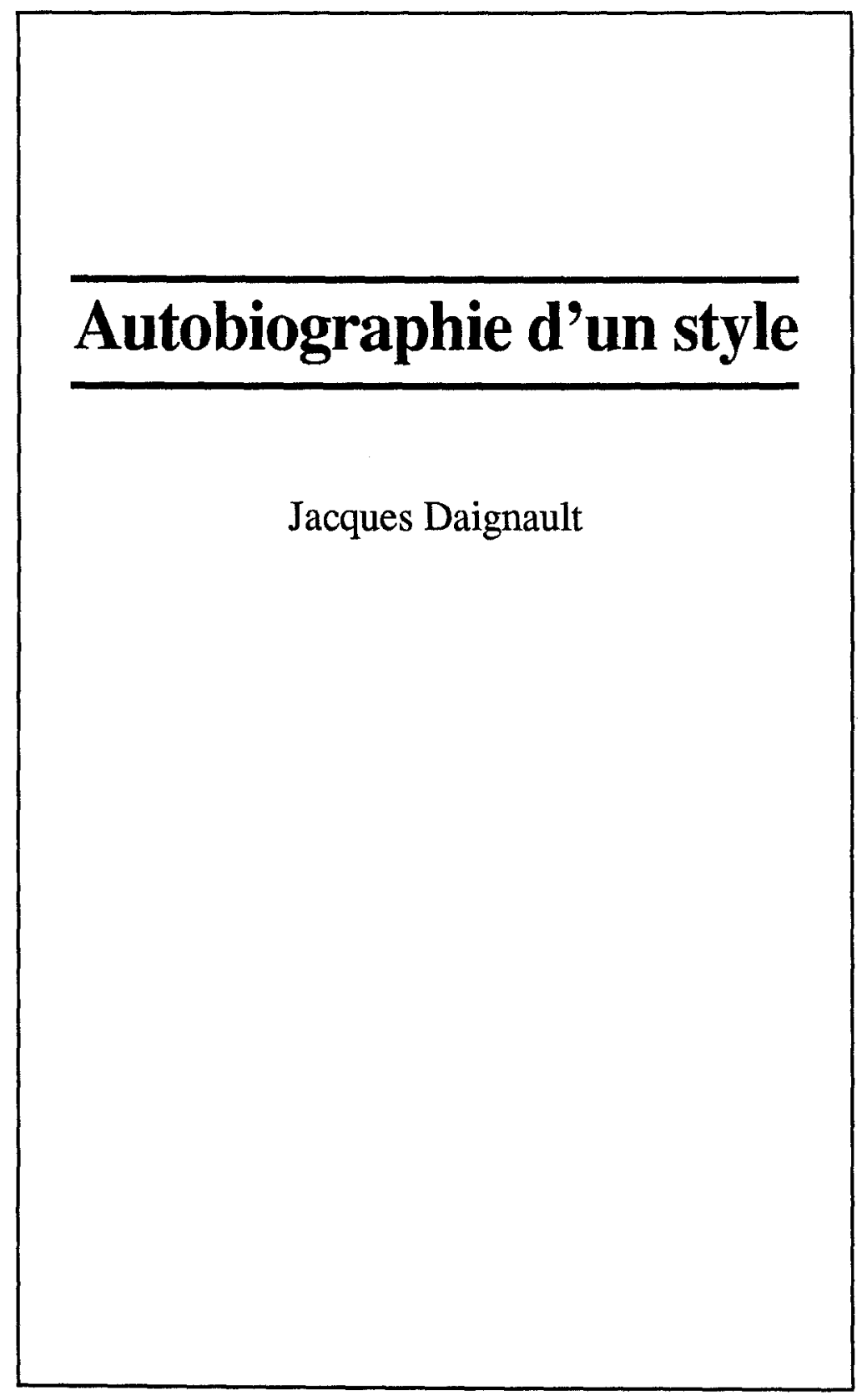




\section{Programme}

\section{Prophétie}

Te me suis déjà cru compositeur. Il y a de cela longtemps. Le dernier argument musical que j'osai écrire avait quelque chose de franchement grec. Mais je ne l'aurai su que plus tard; au moment où je découvrais comment cette pièce constituait un passage: de la musique à la pédagogie. Appelons cela une traversion. Comment l'expliquer?

La répónse insiste quelque part dans la manière dont j'écris; question de style. C'est à peu près cela. Il me faudra toujours revenir à la musique; je veux dire à la manière dont je ne l'ai pas comprise. J'écrivais une mélodie et je la doublais: à la quarte, à la quinte, à l'octave; cela avait manifestement quelque chose de grec. Une curiosité récente - les racines grecques de ma langue - me le confirmait: l'abîme musical qu'exprime le quintieme postulat d'Euclide. 
Épiphanie
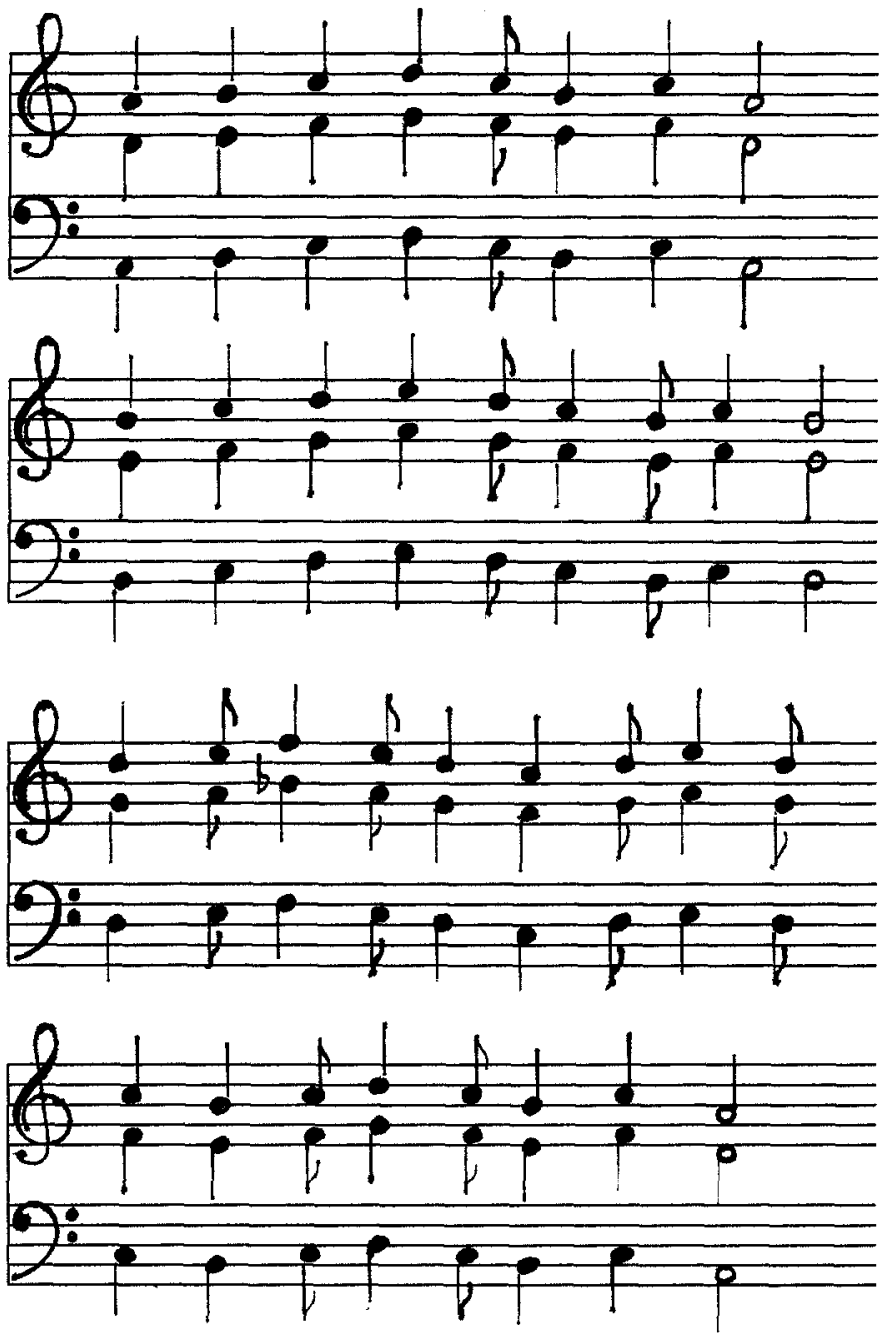


\title{
The Muse
}

\section{Plan de l'Essai}

Pédagogie et stylE oRchestrer a temPo chOrus et écrits

\section{PRatique de l'ÉPochè}

\author{
Présence au sensE \\ aRchéologie d'un scriPt \\ shOt in my llfe \\ PROces(sus) de l'ÉPIgraphe \\ (je) PassE \\ (je me) tRomPe \\ (on a) chOIsi
}




\section{Homo Stylus}

\section{Prologue}

\section{Axiomes}

A1.

- Biographie: enquête autour des événements d'une vie; sutures d'un corps et d'une âme alors autopsiés.

A2.

- Autobiographie désigne [means-names] généralement la biographie de soi.

A3.

- D'après la tradition littéraire française - au moins celle qui va de Buffon à Barthes -le style est la présence de soi dans le texte.

A4.

- J'écris.

Règles

R1.

- Si A1 et A3, alors écrire quelque chose qu'on indexera sous $\mathrm{Cl}$. 
$\mathrm{R} 2$.

- $\mathrm{Si}$ ((A4 et $\mathrm{C} 1)$ et $\mathrm{A} 2)$, alors écrire quelque chose d'autre qu'on indexera sous $\mathrm{C} 2$.

\section{Conjectures}

C1.

- Alors biographie d'un style désignerait [would mean-(nom de bois)] l'enquête autour d'un seul événement: le style. Je veux dire la personnalité de quelqu'un en tant qu'elle est révélée seulement par le style de ses textes. La personnalité jouée au style.

C2.

- Supposons que le style auquel réfere mon enquête soit le mien. Alors biographie d'un style devient autobiographie d'un style.

\section{Question}

\section{Q1.}

- Pourquoi limiter l'enquête sur mes propres sutures, à la seule présence du style dans mes textes?

\section{Epistomac[h]ologie}

\section{Motifs}

M1.

- Je connais des gens, en particulier en éducation, qui n'hésitent pas à condamner mes usages de la langue; on ne dit pas vraiment lesquels. On me reproche simplement de faire obstacle à la compréhension (mutuelle?) des référents, des significations et des autres. Un tel jugement, longtemps $\mathrm{m}$ 'a fait rire. Mais quelque part, vanité et lassitude animaient ma réaction. En dépit 
d'une grande différence de maîtrise dans mes rapports aux deux langues - passablement bonne en français, plutôt cheap en anglais - la manière dont je les traite relève néanmoins d'un même excès: les mots sont des corps dont le sens est mon rapport à l'être. L'épaisseur du mot - et j'essaie d'en prendre soin en français - est matière à sculpter le sens de mon script: écrit; matière analogue aux sons de la langue anglaise (au moins les miens) que ma bouche négocie: au quotidien depuis peu. Je me sens au moins deux fois étranger dans la langue. Cela m'assure - et de manière efficace - de devenir-déjà (d'entrée de jeu; le coup de D) un outsider; ce que je suis toujours dans mon désert curriculaire.

M2.

- MARS 87 (1): Dans la Revue des sciences de l'éducation, au chapitre de la Recension, J.-M. Van der Maren s'en prend au style de Pour une esthétique de la pédagogie. «[...] Je n’ai pas lu l'oeuvre d'un artiste ou d'un romancier, mais celle d'un savant docteur au langage trop savant. [...] Pour une esthétique de la pédagogie est d'une écriture telle que je me demande à qui l'auteur s'adresse. Il n'y a rien là d'esthétique, à moins que l'esthétique ne consiste qu'à réécrire dans une langue de bois ce que d'autres textes av aientrendu accessible.[...] En conclusion, ce livre est digne d'intérêt, même si sa lecture en est pénible. Pourquoi l'artiste de variétés pédagogiques ne s'est-il pas contenté de son art? [...] On peut être efficace, surtout en pédagogie, et l'on peut être académique, même dans une université, sans être obligé d'écrire à la manière de Deleuze et des autres gourous parisiens.»

- MARS 87 (2): Ouf! Il m'invite à publier ma réplique à la suite de son texte.

- MARS 87 (3): L'écriture de cette réplique me dit tout, ou presque, sur la manière confuse dont j'utilise la langue (diffuse serait un bien meilleur mot). «Ici je veux croire Barthes, fût-il un autre gourou parisien: le style est la présence de l'auteur dans le texte. [...] Le malentendu 'est terrible. Je n'ai jamais cru que seuls l'emploi à outrance du je et la description directe de son vécu puissent convenir pour témoigner de soi [en sont-ils même capables!]. On en témoigne tout aussi bien, et peut-être mieux, par ses excès, quand c'est précisément de cela qu'il faut témoigner. Il me fallait excéder le langage parce qu'on m'en 
avait privé! Je n'avais encore rien écrit de plus transparent que cet ouvrage: une autobiographie par le style, de toutes mes frustrations scolaires. [...] c'est en témoignant à l'excès de ma présence subjective [on pourra pas dire que je n'insistais pas!] dans le texte - et par un style excessif, ne l'oublions pas - que j'ai appris, depuis, à penser l'esthétique de la pédagogie dans l'utopie d'une frontière entre, disons, l'objet et le sujet. A qui donc pourrait s'adresser ce livre! [...] aux élèves médiocres qu'on a tenté à plusieurs reprises d'exclure de la culture et qui découvrent à vingt ans seulement - mais avec toutes les maladresses de leur passion - le sens de l'engagement littéraire à mettre en place une écriture pédagogique. Cela prendra peut-être encore des siècles.»

- MARS 87 (4): Je soumets un projet de communication à The Journal of Curriculum Theory Conference, Dayton, Ohio, Bergamo Center, automne 1987.

- AOUT 87: J'essaie de donner un sens au titre proposé de ladite communication: Autobiography of a Style.

- OCTOBRE 87: Je donne ma communication en anglais; comme prévu.

- SEPTEMBRE 88: Je prépare, pour URGENCES, une traversion en français.

\section{Hypothèses (une et demie)}

H1.

- Pinar: «Only as I comprehend the relations among my life history, my biographic present and my intellectual works, am I engaged in educational work.» De mon point de vue l'autobiographie d'un style, ici - cela n'est pas une visée, mais un visa.

$\mathrm{H} 1 / 2$.

- Le style est à la langue, ce que je suis à... (je ne l'ai pas encore trouvé). 


\section{Curriculum}

\section{Procédure}

Écrire en anglais des phrases bien formées.

- Une phrase par ligne.

Intercaler, ça et là, des symboles qui, ensemble, en disent un entier (ou principal) les comprenant tous.

- Ne pas donner le symbole principal en anglais.

Écrire le symbole principal en diagonale et en français:

- le premier mot de la première phrase,

- le deuxième mot de la deuxième phrase,

- le troisième mot de la troisième phrase...

Les mots de la diagonale doivent avoir la même orthographe en français et en anglais;

- mais leur sens doit différer absolument.

- La syntaxe française doit être complètement respectée.

Le sujet de l'épigramme ainsi révélé doit constituer le propos même du présent texte. 
Le titre de 1'épigramme doit être androgyne:

- dans les deux langues: bilingue;

- aux deux niveaux du langage que sont la forme et le sens;

- epicène. Tout un défí!

Le sous-titre doit révéler un nom propre qui né soit pas étrangèr au processus.

\section{Épitomé}

Une double anagramme:

- metheus le PRO, metheus l'ÉPI.

Une double symétrie:

- au plan du tout,

- au plan des parties.

- Une seule exception: la traversion française ne comprend pas de lexique français-anglais; $A d L$ ibitumn'est donc constitué que d'une seule partie: la théorie musicale chez les anciens Grecs.

«La forme coûte chen, disait Valéry. Je ne pouvais en payer le prix en anglais; j'eus donc recours à une double compensation.

- $\tau \varepsilon \chi \vee \eta$ et stylus [retenus].

- Emprunts directs à des encyclopédies et à des dictionnaires [non retenus]. 


\section{Dialogonal(e)}

\section{Procréation}

I CAN'T OR maybe je ne peux pas ne pas être formel.

La la la, is she singing

The chair is out of tune.

She must attend to such a musical abyss.

Rhythm is the son of March.

Thunderbolt echoes back her dire scream.

C.Q.F.D.

\section{Épisode}

7 he flesh is waiting for its statement. Je suis né en mars, me dit ma mère. Je suis d'abord et avant tout le dire ou même l'énoncé de sa chair. Je suis d'abord de peau bien tendue; vibrant encore en sympathie avec le mouvement des vagues d'un tel choc des corps. J'ouis par la peau. J'entends 
les dieux, encore aujourd'hui, distribuant, comme des balles, dons et talents qui frappent à mort les héros tombés sur les champs de bataille. Dans la mémoire des frissons de mon premier dialogue - le cri de ma mème mêlé au mien - ma peau enregistre encore la chaleur du feu, celui de Prométhée: le premier héros encore debout. Pareille méditation transforme littéralement ma quête en symptômes: je suis réellement fiévreux. En finir au plus tôt avec ce texte est une nécessité vitale; cela me rend vraiment malade.

Prométhée a fait l'espèce humaine à son image. Son crime avait quelque chose de généalogique: rendre possible notre destin. «Voici votre monde,» nous dit Prométhée. Ce feu est la mise au point (focus) de l'espèce humaine, le symbole de notre indépendance à l'égard des dieux, le symbole de l'âtre ou du foyer: le nôtre.

Me voici. Je pétris les hommes

A mon image.

Une race qui me ressemble,

Faite pour souffrir, pour pleurer,

Pour goûter le plaisir et la joie.

Et pour te mépriser

Comme moi!

(Goethe in Nietzsche La naissance de la tragédie)

Prométhée: celui qui vient d'abord et qui sait voir au loin: le pré-voyant [fore-thinker]. Nous sommes la prophétie de Prométhée. Notre monde est le théâtre de l'énonciation de sa foi.

La foi de Prométhée est dans la chair du taureau: le transcodage du feu dans la viande.

«Tu as appris aux hommes à travailler et à penser, dit Zeus à Prométhée, mais tu ne leur as pas assez appris à vénérer les dieux, ni à leur offrir des sacrifices, ni à les adorer. [...] Retoume chez 
les hommes, et dis-leur de nous offrir des sacrifices, sinon notre courroux s'abattra sur eux.»

"Les hommes vont offrir des sacrifices aux dieux, " répondit alors Prométhée, «mais il faut que tu viennes toi-même, ô Zeus, choisir ce qu'ils doivent sacrifier.»

Prométhée tua un taureau, cacha la chair dans le cuir du taureau et disposa les entrailles par-dessus. Il fit un autre tas avec les os, mais les recouvrit avec la graisse de telle sorte qu'ils étaient invisibles. Le tas d'os recouvert de graisse était plus gros et plus appétissant. Dès que tout fut prêt, Zeus sentit l'odeur délicieuse du sacrifice préparé et descendit sur terre.

Prométhée vit Zeus et s'exclama:

«O grand Zeus, choisis la part que tu préferes. Celle que toi, roi des dieux, auras choisie sera celle que les mortels continueront à te sacrifier.»

Zeus comprit bien que Prométhée cherchait à le tromper. Pourtant il ne montra pas sa colère, mais choisist délibérément le tas luisant de graisse: les os dénudés apparurent. Par contre, lorsqu'il ôta le cuir du taureau de l'autre tas, la chair fraîche apparut, dégageant son agréable odeur. Depuis ce jour, les hommes sacrifièrent aux dieux la graisse et les os et gardèrent la chair pour eux.

Mais Zeus ne laissa pas impuni cet acte effronté: il décida de priver les hommes du feu, et, si la meilleure part - la chair - leur était réservée, ils devaient désormais la manger crue.

[...]

Prométhée vit quel désastre s'était abattu sur eux; il les prit en pitié et ne les abandonna pas. Sachant que dans le palais de Zeus brillait jour et nuit un feu étincelant, il rampa la nuit jusqu'en haut de l'Olympe vers le palais sacré du dieu suprême. Sans être vu, tout doucement, il prit un peu du feu qui brillait dans la cheminée de Zeus et le cacha dans un bâton creux. Puis, tout joyeux, il s'en retouma chez les hommes avec son précieux larcin.

On connait la suite: Prométhée fut sévèrement puni. Et dans sa collère, Zeus condamna l'humanité à subir éternellement la vengeance des dieux. Héphaiistos, artiste renommé de l'Olympe, modela, sous les ordres de Zeus, la très belle Pandore. Celle-ci fut envoyée sur terre avec pour seule mission de séduire Épiméthée, le frère de Prométhée. Àl'occasion de leurs noces, une boîte - la boîte de Pandore - fut offerte en 
dot. Épiméthée s'empressa de l'ouvrir; en sortirent aussitôt tous les maux qui allaient désormais accabler l'humanité: la faim, la misère, la douleur... Effrayée, Pandore referma la boîte: au moment même où allait en sortir l'espoir.

L'adoration romaine des vaches n'a rien à voir avec la cuisine du taureau. On imagine le nom du cuisinier: Adore, celui ou celle qui ne donne rien aux dieux. Pandore l'instrument de la vengeance des dieux - constituait d'abord une riposte contre la naissance d'Adore.

Nous sommes les descendants de Prométhée et d'Épiméthée; nous devons lutter, sans espoir, contre la vengeance des dieux: cuisiner la chair du monde avec le feu primordial, le Chaos. Crases du feu et de la chair: traversion.

Prométhée est un masque de Dionysos; tous deux symbolisent la douleur de l'enfantement: le cri de ma mère.

In The Birth of Tragedy, Nietzsche told of two gods whom Greek art had workshipped: at first Dionysus, the god of wine and revelry, of ascending life, of.joy and action, of ecstatic emotion and inspiration, of instinct and adventure and dauntless suffering, the god of song and music and dance and drama: - and then, Apollo, the god of peace and leisure and repose, of esthetic emotion and intellectual contemplation, of logical order and philosophic calm, the god of painting and sculpture and epic poetry. The noblest Greek art was a union of the two ideals. In drama Dionysus inspired the chorus, and Apollo the dialogue; the chorus grew directly out of the procession of the satyr-dressed devotees of Dionysus; the dialogue was an after-thought, [c'est moi qui insiste] a reflective appendage to an emotional experience.

Mon premier dialogue - le double cri de ma naissance n'est peut-être que l'écho de la naissance de la Paideia. Épiméthée, celui qui pense après-coup [after-thought], est un masque d'Apollon. Je suis toujours fiévreux, mais confiant; mon propre cri était un cri rempli de joie: non d'espoir. On 
espère jamais que sous le coup d'une intentionnalité. L'espoir est constitutif de l'Histoire: contre l'appréciation immédiate du temps que chacun de nous met à naître; l'espoir enfermé à jamais dans la boîte de Pandore n'est pas le nôtre, mais le signe que nous sommes bel et bien nés. Prométhée a fait l'espèce humaine à son image; Épiméthée a coupé le cordon ombilical. La connaissance et la technique sont des présents de Prométhée; mais la pensée nous vient d'Épiméthée. On y trouverait là le fondement des «humanités» (je ne dis pas «humanisme»); la culture grecque - la Paideia (humanitas, traduisait Virgil) - est faite de ces deux présents-là. 


\section{$\operatorname{Me}[\operatorname{Th}($ ought $)]$}

\section{Prothèse}

Dans la Critique de la faculté de juger, Kant soutient qu'il n'y a pas de méthode (modus logicus) en art mais seulement une manière (modus aestheticus). Cette remarque est peut-être également valable pour l'éducation: il existe peutêtre une méthode d'apprentissage, relativement. à ce qui relève du modus logicus; mais seulement une manière, relativement au modus aestheticus. Cela signifie qu'on n'apprend pas une manière comme on apprend une méthode; il existe un curriculum pour la méthode qui est toujours de méthode (methodus, soutient Kant). Mais un curriculum pour la manière est vraisemblablement une manière; le style, peutêtre, est cette manière de la manière: faire attention au style c'est donner une forme pédagogique au sens, mettre en scène la connaissance. Mais surtout: le style étant la présence de soi dans le texte, mettre en scène la connaissance est l'occasion d'apprendre quelque chose sur le metteur en scène lui-même, s'engager dans le processus éducatif: se penser soi-même; penser quelque chose implique se penser soi-même, c'est ce que penser veut dire [means-names]. Penser n'est pas la même chose que connaître. On peut bien connaître plusieurs choses, mais on ne se connaît jamais soi-même: c'est une 
manière de parler; la connaissance est de méthode. Se connaître soi-même est matièrẹ de pensée et d'écriture (thinkink).

Mettre en scène la connaissance, mettre en scène ce que l'on sait au moyen du style, c'est une manière de penser: autodidactie. Une pensée pédagogique (je ne dis pas didactique) est un pléonasme; quand penser veut dire penser, toute pensée est pédagogique. Pour les Grecs, la Paideia était le curriculum des pensées [méthée $=\mu \eta\rceil \delta \sigma \sigma$ [pensées, desseins] et non $\mu \varepsilon \theta 0 \delta 0 \sigma$ ); non celui des idées ou des concepts. La connaissance est faite de concepts, les croyances sont faites d'idées; la culture est pensée.

En gros, un concept est une rendez-vous entre une catégorie (une forme) et une sensation brute (une matière); une idée est une forme dont la matière est un cadeau des dieux, alors qu'une pensée est une différence qui fait une différence entre le langage dans lequel une idée est collectivement exprimée et le style dans lequel j'exprime la même idée. Une pensée est toujours résiduelle par rapport à une idéologie ou à une connaissance.

Le style est peut-être la forme la plus coûteuse de l'écriture. Le style est toujours autobiographique et autodidactique. Je ne peux m'imaginer travailler sur le style-même dans une activité très théorique - et rester le même; je sentirai toujours au moins le travail de la pensée sur ma peau et dans mon estomac. Ce que nous appelons inspiration n'est pour moi qu'une somatisation de l'écriture. J'ai grandement besoin d'epistomac[h]ologie.

Dans ce texte, il n'y a pas de notes biographiques directes; le style est ma manière préférée. 


\section{Épithète}

\section{Qu'est-ce qu'un argument?}

En mathématique.

- Un argument quantitatif: une certaine valeur d'après laquelle une fonction est calculée. La variable indépendante d'une fonction (l'indépendance signifie et ne signifie pas l'arbitraire, question d'épistémologie).

En rhétorique.

- Un argument qualitatif: un style au moyen duquel un discours est mis en forme. L'indépendance littéraire de la pensée (l'indépendance fonde ou réfute le libre arbitre, question d'ontologie). 


\section{Ad infinitum}

\section{Prouver}

\section{La diagonale de Cantor}

P $\mathrm{n}$ voici une version simplifiée (traduction commentée de Dla version de Davis et Hersh). Soient toutes les fonctions $f$ définies sur les entiers $1,2,3, \ldots$. [on pourrait imaginer plus simplement l'expression de tous les nombres réels]. Théoreme: Il n'est pas possible de dresser la liste de toutes ces fonctions [impossible d'assigner à chaque nombre réel un nombre entier (au sens d'un index)]. Preuve: Supposons que cela soit possible. Il existerait alors une première fonction dans la liste. Appelons-la $f_{1}$. Il existerait une deuxième fonction $f_{2}$, etc. Maintenant, pour chaque nombre $n$, où $n$ prend [successivement] les valeurs $1,2,3, \ldots$, examinons les nombres $f_{\mathrm{n}}(n)+1$ [c'est là une façon d'exprimer la diagonale: la première valeur de la première fonction +1 , la deuxième valeur de la deuxième fonction +1 , etc.]. Cette suite de nombres constitue elle-même une fonction définie sur les entiers, elle doit donc, en vertu de ce que l'on a supposé, se retrouver dans la liste. Appelons-la $f_{\mathrm{k}}$. Par définition $f_{\mathrm{k}}(n)=$ $f_{n}(n)+1$, et cela vaut pour $n=1,2,3, \ldots$ Cela vaut en par- 
ticulier pour $n=k$; cela conduit donc à $f_{\mathrm{k}}(k)=f_{\mathrm{k}}(k)+1$. Ainsi, $0=1$ : une contradiction.

En conclusion, l'infini des nombres réels est «plus grand» que l'infini des nombres entiers.

Corollaire: le discours ne peut pas indexer le langage; penser est donc infiniment possible.

\section{PI}

PensEr à trois paragraphes, exactement. Dans le premier, il y a quatre phrases. Dans la première phrase, je compte cinq mots. Dans cette phrase d'ouverture, le neuvième mot - qui ne peut pas être là - est fait de deux lettres.

Dans ce mot irrationnel, le sixième signe - qui peut être aisément pensé - suggère la cinquième lettre de l'alphabet, celle que le sous-titre de ces paragraphes laisse en blanc: pour mieux y penser. Penser, en effet, à ce mot de trois lettres - le sous-titre - qui commence par la cinquième lettre de ces trois paragraphes...

\section{$3.1415926535 \ldots$}




\section{Ad libitum}

\section{Théorie musicale grecque}

T'homophonie - une simple ligne mélodique - constitue le $L_{\text {trait dominant de la musique grecque ancienne. Quand }}$ des voies étaient superposées, elles l'étaient à l'unisson ou à l'octave; parmi les intervalles inférieurs à l'octave, seules la quarte et la quinte étaient considérées comme consonnantes.

La théorie des gammes de la Grèce antique, telle que nous la connaissons aujourd'hui, est surtout le fruit d'Aristoxène (4 siècles avant J.-C.). L'étendue des sons était conçue comme une ligne le long de laquelle les différentes hauteurs correspondaient à des points. La distance entre deux points s'appelaient un intervalle (diastema); un groupe d'intervalles successifs constituait un sytème (systema). Tout composé d'intervalles constituait un système particulier (deux intervalles) ou général (plus de deux intervalles). Parmi les sytèmes généraux, on en retient généralement trois: le Sytème Parfait le plus Grand (systema perfectum maximum), le Système Parfait le plus Petit (systema perfectum minimum) et la réunion des deux dans le Système Immuable (systema immutatum). 
La mélodie de l'Épiphanie est écrite dans le Système Parfait le plus Grand. En la doublant à la quinte inférieure, il me fallait recourir au tétracorde synnemenon (tétracorde final du Système Parfait le plus Petit) et donc «réalisen» le Système Immuable. Faut-il dire que je n'en étais pas conscient?

\section{S.P.G.}

- LA Proslambanomenos

- SI Hypate Hypaton

- Ut Parhypate Hypaton

- Ré Lichanos Hypaton (Rousseau: Hypathon Diatonos)

- Mi Hypate meson

- Fa Parhypate meson

- Sol Lichanos Meson (Rous: Meson diatonos)

- La Mese

- Si Paramese

- Do' Trite Diezeugmenon

- Ré' Paranete Diezeugmenon (Rous: Diezeugmenon Dianotos)

- Mi’ Nete Diezeugmenon

- Fa' Trite Hyperbolaion

- Sol' Paranete Hyperbolaion (Rous: Hyperboléon Dianotos)

- La' Nete Hyperbolaion

\section{S.P.P.}

- LA SI Do Ré Mi Fa Sol La Sib Do' Ré’

La prosodie finale «est» [IS:Immutable System] épiphanique. 


\title{
Mets (Heu!)
}

\author{
E \\ $P \quad P$ \\ BIBLIOGRAPHY \\ O
}

Bailly, A.,Dictionnaire Grec-français, Paris, Hachette, 1950.

Barthes, R., Le degré zéro de l'écriture, Paris, Seuil, 1972.

Blom, E., (Ed.) Grove's Dictionary of Music and Musicians, London, MacMillan \& Co. Ltd, 3 Vol., 1966.

Chantraine, $\mathrm{P} .$, Dictionnaire étymologique de la langue grecque. Histoire des mots, Paris, Klincksieck, 1970.

Davis, P.H., Hersh, R., The Mathematical Experience, Boston, Birhäuser, 1981.

Ducrot, O., Todorov, T., Dictionnaire encyclopédique des sciences du langage, Paris, Seuil, 1972.

Durant, W., The Story of Philosophy, New York, Garden City, 1926.

Kant, I., Critique de la faculté de juger (trad. A. Philonenko), Paris, Vrin, 1984. 
Marrou, H.-I., Histoire de l' éducation dans l' antiquité, Paris, Seuil, 1948.

Nietzsche, F., La naissance de la tragédie (trad. G. Bianquis), Paris, Gallimard, 1949.

Petiska, E., Sklenar, Z., Mythes et légendes de la Grèce antique (trad. A. Gründ), Paris, Gründ, 1980.

Pinar, W.F., «Life History and Educational Experience: Part Two», Journal of Curriculum Theorizing, Rochester, 3:1, 1981.

Revue des sciences de l'éducation, Montréal, XIII, 1, 1987.

Rousseau, J.-J., «Dictionnaire de musique» in Oeuvres complètes, Paris, Dupont, Vol. 12-13, 1824. 


\section{Epigramme}

\section{Prosodie}

Au quai long vie de la-lu-ré

À lire aussi le long de la-lé

Vous étiez absents

Au rendez-vous, gens

Avez-vous peur des enfants

\section{Épigraphe}

$\Pi^{{ }^{*} \text { ide }_{\mathrm{ia}}}$ 